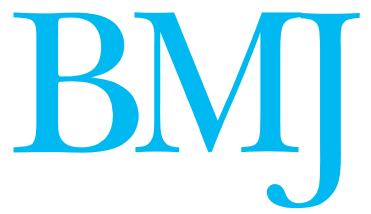

\title{
Placebos in practice
}

\author{
Doctors use them, they work in some conditions, but we don't know how they work
}

Papers p 944
$\mathrm{P}$ lacebo comes from the Latin for "I will please." Pleasing a patient would seem to be a good thing to do. Yet considerable controversy exists about the use of a biologically inert or irrelevant substance with therapeutic intent. Nitsan and Lichtenberg show in this issue (p 944) that placebos are often used in modern medicine. ${ }^{1}$ Their survey of 89 doctors and nurses providing hospital based and ambulatory care in Israel found that $60 \%$ used placebos in their practice, most often (43\%) to fend off an "unjustified" demand for medication, to calm a patient (38\%), as an analgesic (38\%), or, more problematically, as a diagnostic tool $(28 \%)$. The paper makes it clear that the placebo pleases modern doctors. Should it? If the placebo effect is real, is it right to use it?

Most of medicine used placebos at one time. Medicine in the 20th century was supposed to end this. We would use only scientifically proved active pharmacological and surgical interventions. Yet only about half of medical treatments are supported by evidence. ${ }^{2}$

A conference held at the National Institutes of Health in the United States in 2002 reviewed the evidence and concluded that we needed more science, not fewer placebos. ${ }^{3}$ It called for research on brain and body pathways that mediate placebo effects, and for optimising the use of the placebo phenomenon while attending to ethical and practical concerns about it. The response to placebos varies widely across different cultures, but the myth that placebo responders are uneducated, unintelligent, free of serious medical illness, or histrionic was not supported by current research. ${ }^{\mathrm{w1}}$ The conference also noted the importance of the nocebo effect-negative expectations can produce negative results. ${ }^{\text {w2 }}$ In the 19 th century, for example, tomatoes were believed to be poisonous, and many people were treated in hospitals for symptoms of tomato poisoning." ${ }^{\text {3 }}$

Doubts have been raised about the usefulness of the placebo in conditions other than pain. A recent meta-analysis and related Cochrane report found little evidence that using a placebo improved symptoms, with the exception of pain relief. ${ }^{45}$ This meta-analysis had numerous problems. ${ }^{6}$ It lumped together 40 heterogeneous outcome assessments and further restricted the power to detect an effect by dichotomising them as improved or not, rather than treating these outcomes as continuous variables. The one analysis that did treat outcome continuously found a significant effect-reduction in pain. What this meta-analysis really showed is not that the placebo doesn't cure anything, but rather that it does not cure everything.

Despite such scientific scepticism, the placebo phenomenon seems to influence the behaviour of patients and doctors. The growth of patients' interest in integrative or holistic medicine in the past decade is perhaps a reaction to the growth of evidence based medicine. ${ }^{78}$ Most placebos are relatively harmless. Modern medicine involves treatments, such as surgery, chemotherapy, and bone marrow transplantation, that are effective but also toxic. Many patients may choose integrative medicine as a kinder and gentler treatment that harnesses rather than eschews the placebo effect and engages them as participants in their care, especially in the treatment of chronic problems such as anxiety and pain that are often not well managed in

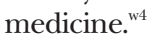

That an idea, feeling, or relationship can have a real effect on the body is now established. Scientific domains such as psychoneuroimmunology and psychoneuroendocrinology are helping us to understand mechanisms whereby belief in benefit might affect resistance to disease, for example as a form of stress reduction with physiological consequences. Considerable evidence indicates that depression, for example, affects outcome in heart disease and cancer. ${ }^{9} 10$

In the survey by Nitsan and Lichtenberg, few doctors $(15 \%)$ used placebos without deception. ${ }^{1}$ However, deception is not a necessary component of the placebo response. Many have justifiable ethical concerns about deliberately deceiving patients regarding the nature of their treatment. But perhaps therapeutic intent and expectation are sufficient to mobilise healing in patients. ${ }^{\mathrm{w}}$

The use of the placebo as a diagnostic tool by $28 \%$ of respondents is more troubling, as the authors note. That a patient gets pain relief from a placebo does not imply that the pain is not real or organic in origin. Ample evidence shows that psychological interventions such as hypnosis can alter the perception of pain dramatically. Beecher noted that grievously wounded soldiers on the Anzio beachhead required less analgesia than less seriously injured patients in Boston. ${ }^{11}$ Their intense desire to survive overrode their focus on the pain of their injuries. Psychological factors such as redirected attention, distraction, and changes in perceived meaning of real pain can alter its intensity.

References w1 to w5 are on bmj.com 
Thus the use of the placebo for "diagnosis" of whether or not pain is real is misguided.

The placebo effect, thought of as the result of the inert pill, can be better understood as an effect of the relationship between doctor and patient. ${ }^{12}$ Adding the doctor's caring to medical care affects the patient's experience of treatment, reduces pain, and may affect outcome. This survey makes it clear that doctors continue to use placebos, and most think they help. We cannot afford to dispense with any treatment that works, even if we are not certain how it does.

\section{David Spiegel Willson professor}

Stanford Medical Center, Stanford, CA 94305-5718, USA (dspiegel@stanford.edu)

Competing interests: None declared.

1 Nitzan U, Lichtenberg P. Questionnaire survey on use of placebo. BMJ 2004:329:944-6.

2 Hardern RD, Leong FT, Page AV, Shepherd M, Teoh RC. How evidence based are therapeutic decisions taken on a medical admissions unit? Emerg Med J 2003;20:447-8.
3 Kleinman A, Guess H, Wilentz JS. An overview. In: Guess H, Kleinman A, Kusek J, Engle LW, ed. The science of the placebo. London: BMJ Books, 2002:1-32.

4 Hrobjartsson A, Gotzsche PC. Is the placebo powerless? An analysis of clinical trials comparing placebo with no treatment. $N$ Engl J Med 2001;344:1594-602.

5 Hrobjartsson A, Gotzsche P. Placebo interventions for all clinical conditions. Cochrane Database Syst Rev 2004:3:CD003974.

6 Spiegel D, Kraemer H, Carlson R. Is the placebo powerless? [letter]. $N$ Engl J Med 2001;345:1276.

7 Barnes PM, Powell-Griner E, McFann K, Nahin RL. Complementary and alternative medicine use among adults: United States, 2002. Hyattsville, MD: National Center for Health Statistics, 2004. (Advance data from vital and health statistics; No 343 .)

8 Vozeh S. Is the increasing use of evidence-based pharmacotherapy causing the renaissance of complementary medicine? Br J Clin Pharmacol 2003;56:292-6.

9 Grippo AJ, Johnson AK. Biological mechanisms in the relationship between depression and heart disease. Neurosci Biobehav Rev 2002:26:941-62.

10 Spiegel D, Giese-Davis J. Depression and cancer: mechanisms and disease progression. Bioll Psychiatry 2003;54:269-82.

11 Beecher HK. Relationship of significance of wound to pain experiences. JAMA 1956;161:1609-13.

12 Brody $\mathrm{H}$. The doctor as therapeutic agent: a placebo effect research agenda. In: Harrington A, editor. The placebo effect: an interdisciplinary exploration. Cambridge: Harvard University Press, 1997:77-92.

\section{What causes chronic fatigue syndrome?}

\section{Infections, physical inactivity, and enhanced interoception may all play a part}

C hronic fatigue syndrome, also known as myalgic encephalomyelitis, is an illness of unknown nature and cause, but most medical authorities now accept its existence. ${ }^{1-3}$ Research about its cause has been hampered by the absence of a biological marker, the heterogeneous nature of the illness, and difficulties in differentiating cause from effect. ${ }^{23}$ Yet, some progress has been made, particularly when causes are divided into predisposing, triggering, and maintaining factors.

Women get chronic fatigue syndrome more commonly than men for unknown reasons, although increasing evidence suggests a genetic influence on the illness. ${ }^{13}$ Premorbid mood disorders are replicated risk markers for chronic fatigue syndrome; ${ }^{13}$ the risks may be inflated by shared symptoms or they may be markers for those patients with comorbid mood disorders. $^{13-5}$ Another replicated premorbid risk marker is increased consulting of a doctor for minor illnesses up to 15 years before diagnosis, ${ }^{\mathrm{w} 1 \mathrm{w} 2}$ suggesting a general vulnerability for either ill health or seeking health care, the latter possibly being mediated by comorbid anxiety. ${ }^{4}$

Certain infectious illnesses, such as Epstein-Barr virus, $\mathrm{Q}$ fever, and viral meningitis, can trigger chronic fatigue syndrome, ${ }^{\text {3.-w8 }}$ common upper respiratory infections do not. ${ }^{\text {w3 }}{ }^{\mathrm{w} 9}$ Little evidence exists of persistent infection in patients with chronic fatigue syndrome. ${ }^{\text {w10 }}$ An immune cause has not been established, ${ }^{6}$ although preliminary research suggests that immune responses to exercise in these patients may be abnormal. ${ }^{\mathrm{w} 11}$ The symptoms of chronic fatigue syndrome are similar to the symptoms and consequent behaviour of people with acute infection. ${ }^{2 \text { w12 }} \mathrm{A}$ low cortisol level has repeatedly been found to be associated with chronic fatigue syndrome, and recent research suggests this may be secondary to the physical inactivity and sleep disturbance found with long standing chronic fatigue syndrome. ${ }^{7}$ w13 Illness maintaining factors may include illness beliefs that encourage avoidant coping. ${ }^{\mathrm{w} 14 \mathrm{w} 15}$

Viner and Hotopf publish in this issue a 30 year cohort study of 16567 babies born in 1970 (p 941), in which they report childhood predictions of self reported chronic fatigue syndrome and myalgic encephalomyelitis developing in adulthood. ${ }^{8}$ Their most important findings were that chronic fatigue syndrome was predicted by having a disabling illness in childhood and never or hardly ever doing sport out of school at age 10. Premorbid psychological distress in either the mother or the child did not predict the illness.

Previous healthcare attendance for ill health does seem to predict corroborated chronic fatigue syndrome. $^{w_{1}}{ }^{\text {w2 }}$ Physical ill health in childhood seems to predispose to medically unexplained ill health in adulthood generally only when the childhood diagnosis is uncertain rather than established. ${ }^{\text {w16 }}$ Neither the mother's nor the child's or adolescent's psychological distress predicted chronic fatigue syndrome, a finding that contrasts with most studies of both children and adults developing the illness. ${ }^{\mathrm{w} 17 \mathrm{w} 18}$ Data from Viner and Hotopf's study may be more reliable, having been gathered contemporaneously. We need to learn how certain childhood illnesses predispose people to later chronic fatigue syndrome.

The novel finding was that 10 year old children who were reported by their mothers to "never or hardly ever" play sport in their spare time had twice the risk of chronic fatigue syndrome in adulthood. In contrast, school sport had no effect; this might be explained by the compulsory nature of some school sport. Although statistically significant being more sedentary was not a strong risk marker, since $84 \%$ of those

Papers p 941 